\title{
A Multidisciplinary Approach for Restoring Function and Esthetics in a Patient with Worn out Dentition: a Clinical Report
}

\author{
Authors \\ Dr Ruby $^{1}$, Dr Manish Kumar ${ }^{2}$, Dr Hanish Chaudhary ${ }^{3}$ \\ ${ }^{1}$ Post Graduate student, I.T.S. Dental College, Muradnagar \\ ${ }^{2}$ Reader, Department of Prosthodontics, Mahatma Gandhi Dental College, Jaipur \\ ${ }^{3}$ Post Graduate Student, I.T.S. Dental College, Muradnagar
}

\begin{abstract}
Cases of severe attrition and abrasion are common finding in daily clinical practice. This leads to loss of vertical dimension, chewing inefficiency and poor esthetics. These cases require complete oral rehabilitation which can be successfully achieved by a systematic approach integrating various concepts of prosthodontics. Full mouth rehabilitation cases are one of the most difficult cases to manage. They involve not only the replacement of the lost tooth structure but also restoring the lost vertical dimension. Patients generally present with isolated problems in individual tooth, or in isolated teeth in different locations in the oral cavity. Very rarely, patients will present with total wear and tear of all the teeth. This article describes the management of a case with severely attrited, abraded and worn out dentition.

Key Words: Attrition; abrasion; full mouth rehabilitation; loss of vertical dimension; occlusal splint; tooth loss; mutilated dentition
\end{abstract}

\section{INTRODUCTION}

Attrition is the loss of tooth structure by mechanical forces from opposing teeth. Abrasion is the pathological wearing of tooth substance through some abnormal mechanical process. It initially affects the enamel and, if left unchecked, may proceed to the underlying dentine. Once past the enamel, attrition quickly destroys the softer dentine. Functional habits such as chewing and swallowing usually put very little force on opposing teeth. Para functional habits, such as clenching and clicking the teeth together nervously, place greater amount of forces on opposing teeth and begin to wear the teeth. As expected, wear usually begins on the incisal or occlusal surfaces. The restorative implications of tooth wear are often complicated by the age of the patient, para-functional habits, compromise of conventional fixed restorations and the lack of inter-occlusal space. Dentists are blessed with the ability to change someone's self-esteem, confidence and possibly the course of life. ${ }^{[1]}$ Patient with tooth wear may require restorative procedures to achieve appropriate function, esthetics and comfort. Occlusal rehabilitation requires a correlation of biological factors, mechanical principles and esthetic requirements with treatment procedures. Excessive occlusal 
wear may manifest itself in a reduction of the vertical dimension of occlusion. Restored vertical dimension is based on the concept that by providing the patient with an interocclusal splint designed to restore the previously lost occlusal vertical dimension; all abnormal muscle activities will be eliminated or reduced ${ }^{[2-7]}$ A combination of new generation materials along with improved clinical procedures work in tandem to produce an esthetic, long lasting and functional outcome, satisfying both the clinician as well as the patient.

\section{CASE REPORT}

A 38-year-old male patient presented to the Department of endodontics of the I.T.S. Dental College \& Research Institute in muradnagar, ghaziabad, India, with severe attrition and abrasion of all of his teeth. He was very selfconscious about his appearance. A detailed history revealed patient had the habit of bruxism and brushing his teeth vigorously with hard bristle tooth brush. Extra oral examination revealed an unaesthetic smile. The intraoral examination (Fig. 1) revealed a Class I malocclusion with the surfaces of the teeth were rough with the enamel either not visible or very thin and hypoplastic over the crowns of all teeth and yellow-brown in color. The dentin, where it was exposed, was brown and hypersensitive. The patient had all the permanent teeth erupted including third molars. Tooth 36 was completely decayed with total loss of clinical crown. Tooth 41 had Miller's Grade II gingival recession ${ }^{[8]}$ with marginal gingiva extending upto mucogingival junction. The panaromic radiograph revealed that the teeth had normal pulpal morphology while the enamel appeared to be very thin or missing on all the teeth.

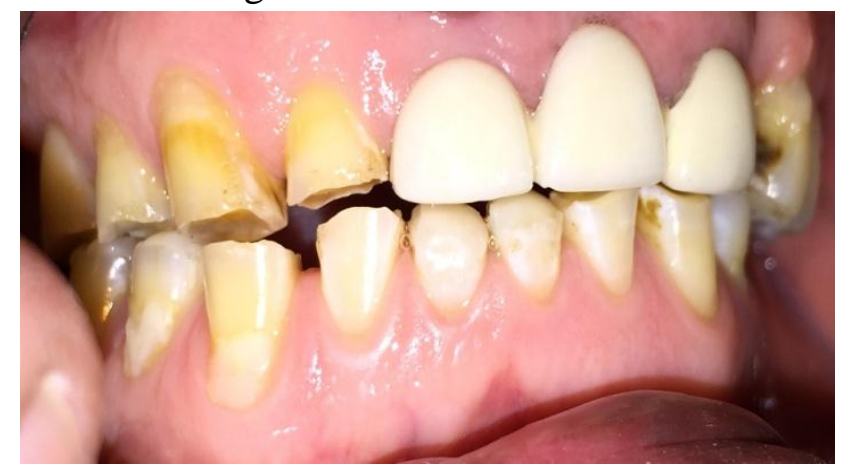

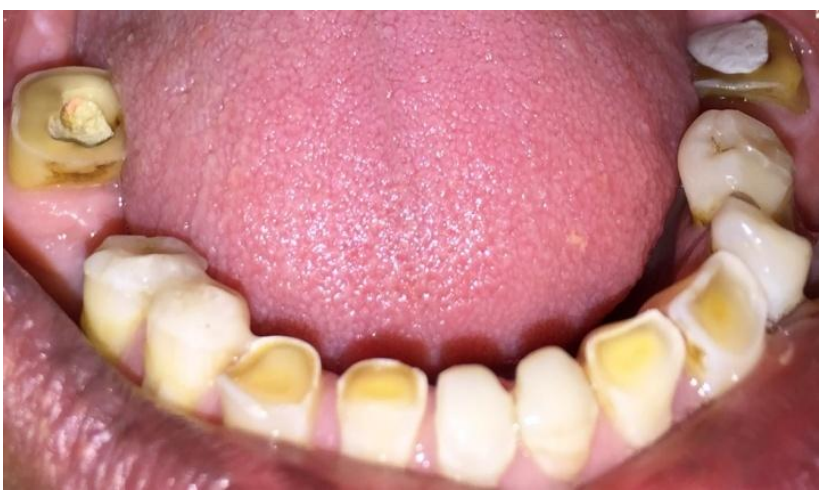

Fig. 1: Pre-treatment intra-oral photographs

\section{Treatment Plan}

The treatment plan involved an interdisciplinary approach consisting of extraction of root stump, periodontal plastic surgery for the tooth with gingival recession, endodontic treatment followed by the fabrication of metal-ceramic crowns for maxillary and mandibular teeth by a prosthodontist. The treatment objectives included the following: Crown restorations to reduce the reported sensitivity of the teeth, improve the esthetics, and restore masticatory function.

\section{TREATMENT}

Extraction of grossly decayed 36 and 46 was carried out. After a thorough oral prophylaxis, periodontal plastic surgery using a free connective tissue graft harvested from Premolar-Molar region of hard palate was carried out in 41 for correction of gingival recession in the Department of Periodontolgy and Oral Implantology. Intentional root canal treatment was carried out from 2nd premolar to 2nd premolar for both maxillary and mandibular arch in the Department of Conservative Dentistry and Endodontics. The vertical dimension of the patient was determined and his 5 to $6 \mathrm{~mm}$ of interocclusal space needed to be modified. An occlusal splint with an increased vertical dimension of $3 \mathrm{~mm}$ was placed for one month in order to assess the acceptability of the new vertical dimension. The patient was asymptomatic and tolerated the new vertical dimension well. Diagnostic casts were made, as were face-bow and protrusive records. Casts were mounted in centric relation in a semi-adjustable 


\section{JMSCR Vol||3||Issue||12||Page 8659-8662||December}

articulator $\left(\mathrm{Hanau}^{\mathrm{TM}}\right.$ Wide-Vue Arcon Articulator, Waterpik, Fort Collins, USA). A diagnostic wax up was done and the articulator was set for condylar and incisal guidance. Later, preparations of all the teeth were done to receive metal-ceramic restorations. Impressions were taken using polyvinyl-siloxane impression material (ReprosilR; Dentsply Caulk, Dentsply International Inc.) using custom trays and interocclusal relationships were recorded. The vertical dimension was increased by $3 \mathrm{~mm}$ in the premolar region in order to restore a favorable occlusal relationship. All prepared teeth were restored with provisional crowns cemented with Provicol (Voco GmbH, Germany). The occlusal records were transferred to a semi- adjustable articulator with a facebow, and the final casts were mounted. Keeping in mind less occlusal clearance in molar region, it was decided to fabricate cast crowns for mandibular right and left 2nd and 3rd molars. Metal frameworks were fabricated and were evaluated intraorally to determine the marginal fit, followed by the ceramic firing. Prior to glazing of the ceramic material a trial insertion was performed, again to enable final occlusal refinement. The patient's natural occlusal scheme (canine-protected occlusion) and anterior guidance were preserved in the definitive restorations to decrease lateral forces on the posterior dentition. The crowns were then completed in the laboratory and cemented with glass ionomer cement (GC, luting \& lining cement, GC Corporation, Tokyo, Japan) (Fig. 4). The anterior and posterior metal-ceramic crowns were satisfactory both esthetically and functionally. The patient was highly satisfied with the treatment results.

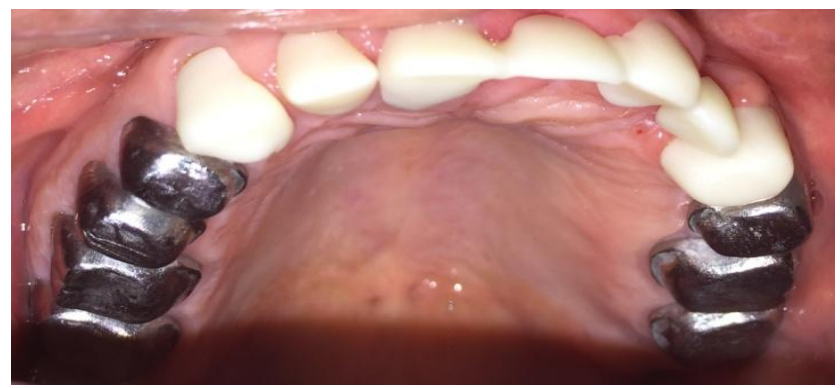

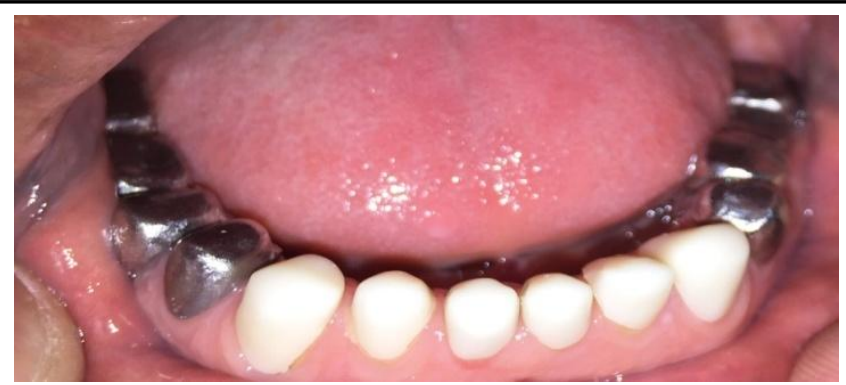

Fig. 2: Copings in place

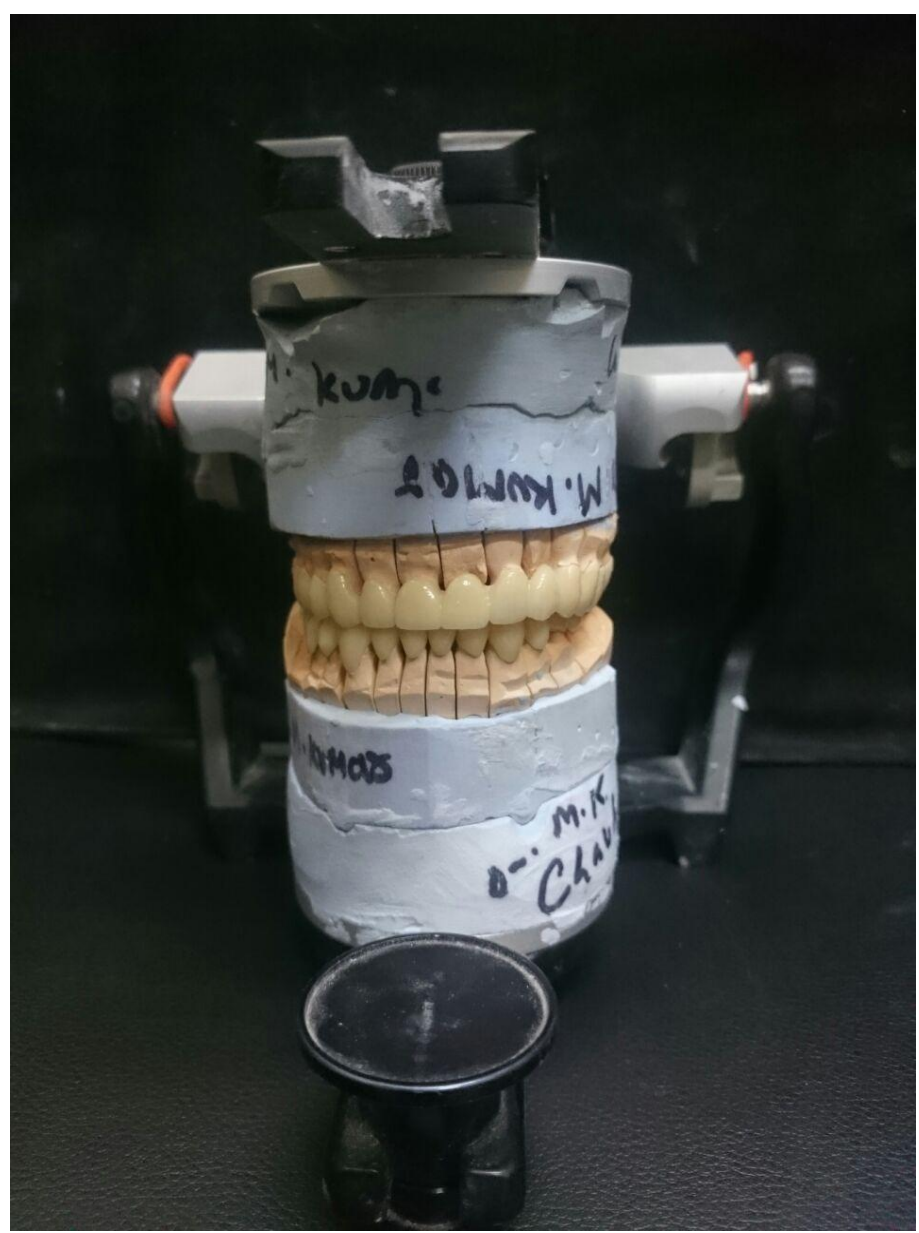

Fig. 3: Ceramic Build up on articulator

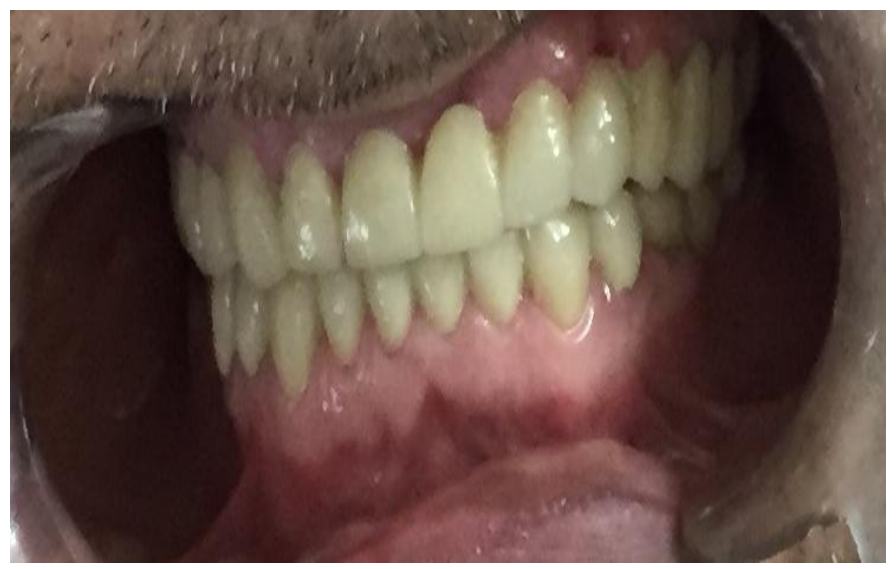




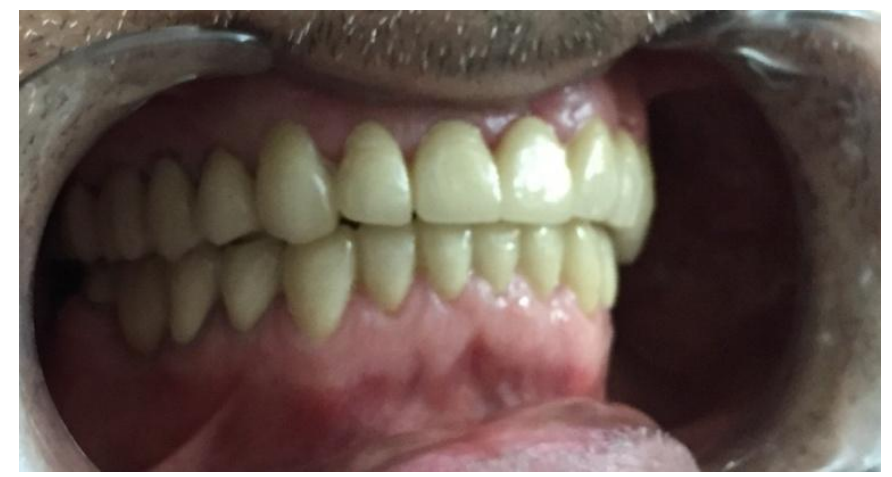

Fig. 4: Post treatment intra-oral photographs

\section{DISCUSSION}

The employment of all the diagnostic, therapeutic and restorative procedures available for the treatment and prevention of dental diseases is referred to as 'Full Mouth Rehabilitation' ${ }^{\text {[9] }}$ The primary aim of the process of mouth rehabilitation is to restore the functional and structural integrity of dental arches. The clinician must plan the treatment diligently, taking into account the need of altering the vertical dimensions and the type of occlusal scheme to be incorporated. Treatment plan is designed to meet biologic, restorative and esthetic requirements. Several materials are available to the dental practitioner as far as restorative modalities are concerned. However several limitations may exist. For instance in the present patient, we could use ceramic laminates to restore esthetics as metal- ceramic crowns require more aggressive removal of tooth structure. But ceramic laminates fail to raise vertical dimension and restore proper occlusal intercuspation. Also laminates cannot withstand high masticatory loads. Though All-ceramic crowns offer better esthetics they fail to resist high masticatory loads and also require more extensive tooth preparation. Therefore metal-ceramic crowns were chosen. Canine protected occlusal scheme was adopted as it prevents destructive occlusal forces on posterior teeth. $^{[10]}$

\section{CONCLUSION}

An important aspect of restoring worn out teeth is the protection of remaining tooth structure. Teeth already weakened by loss of large amount of tooth structure are ill-equipped to withstand occlusal forces. Protection can be best provided by restoring them with the crowns which further restrict their wear and tear. In this patient, enhanced esthetics and improved function was possible because the clinical crowns and root forms were favorable for complete coverage restorations. The post-operative follow up revealed meticulous hygiene maintenance by the patient.

\section{REFERENCES}

1. Binkley CJ, Binkley TK. A practical approach to full mouth rehabilitation. J Prosthet Dent. 1987;57:261-5.

2. Schweitzer JM. An evaluation of 50 years of reconstructive dentistry, Part I: jaw relations and occlusion. J Prosthet Dent. 1981;45:3838.

3. Schweitzer JM. An evaluation of 50 years of reconstructive dentistry, Part II: Effectiveness. J Prosthet Dent. 1981;45:49298.

4. Goldman I. The goal of full mouth rehabilitation. J Prosthet Dent. 1952;2:24651.

5. Bronstein BR. Rationale and technique of biomechanical occlusal rehabilitation. J Prosthet Dent. 1954;4:352-67.

6. Schweitzer JM. A conservative approach to oral rehabilitation. J Prosthet Dent. 1961;11:119-23.

7. Abrahamsen TC. The worn dentitionpathognomonic patterns of abrasion and erosion. Int Dent J. 2005;4:268-76.

8. Miller PD Jr. A classification of marginal tissue recession. Int J Periodont Restor Dent. 1985;5:9.

9. Landa JS. An analysis of current practices in mouth rehabilitation. J Prosthet Dent. 1955;5:527-37.

10. D'Amico A. functional occlusion of natural teeth in men. J Prosthet Dent. 1961;11:899915 . 